УДК 517.9

\author{
T. I. Vdovenko, M. E. Dudkin
}

\title{
DUAL PAIR OF EIGENVALUES IN RANK ONE SINGULAR PERTURBATIONS
}

T. I. Vdovenko, M. E. Dudkin. Dual pair of eigenvalues in rank one singular nonsymmetric perturbations, Mat. Stud. 48 (2017), 156-164.

In the separable Hilbert space, we discuss the eigenvalue problem for a rank one singular nonselfadjoint perturbation of a selfadjoint operator $A$, by nonsymmetric potential $\left(\delta_{1} \neq \delta_{2}\right)$ in the form $\tilde{A}=A+\alpha\left\langle\cdot, \delta_{1}\right\rangle \delta_{2}$. We give the constructive description of such sort operator $\tilde{A}$ which possess two new points in the point spectrum in case of weakly singular perturbations.

1. Introduction. There are many publications (see [1]-[5] and references) devoted to the constructive description and investigations of spectral properties of the formal expression $-\Delta+\alpha \delta\left(x-x_{0}\right)$, where $\Delta=\frac{d^{2}}{d x^{2}}$ is the Laplace operator perturbed by $\delta\left(x-x_{0}\right)$-potential concentrated at the point $x_{0} \in \mathbb{R}$ and $\alpha \in \mathbb{R}$ is a coupling constant.

The formal expression $-\Delta+\alpha \delta\left(x-x_{0}\right)$ has more precise sense of a singular self-adjoint perturbation $([1]-[5],[7]-[10],[18,19])$ in the form $-\tilde{\Delta}:=-\Delta+\alpha\left\langle\cdot, \delta\left(x-x_{0}\right)\right\rangle \delta\left(x-x_{0}\right)$, where $\alpha \in \mathbb{R} \cup \infty, \delta\left(x-x_{0}\right) \in \mathcal{H}_{-2}:=W_{2}^{-2}\left(\mathbb{R}^{1}\right)$ is a negative Sobolev space corresponding to $W_{2}^{2}\left(\mathbb{R}^{1}\right)$ and $\langle\cdot, \cdot\rangle$ is the dot product for $W_{2}^{2}\left(\mathbb{R}^{1}\right)$ and $W_{2}^{-2}\left(\mathbb{R}^{1}\right)$. The operator $-\tilde{\Delta}$ acts as follows:

$$
-\tilde{\Delta} f=-f^{\prime \prime}+\alpha f\left(x_{0}\right) \delta\left(x-x_{0}\right),
$$

for $f \in \mathfrak{D}(\tilde{\Delta})=\left\{f \in W_{2}^{1}(\mathbb{R}) \cap W_{2}^{2}\left(\mathbb{R} \backslash\left\{x_{0}\right\}\right) \mid f^{\prime}\left(x_{0}+\right)-f^{\prime}\left(x_{0}-\right)=\alpha f\left(x_{0}\right)\right\}([1,5])$.

In this article we consider some generalization of expression (1) in the form:

$$
\begin{gathered}
-\tilde{\Delta} f=-\Delta f+\alpha f\left(x_{1}\right) \delta\left(x-x_{2}\right), \quad x_{1} \neq x_{2}, \quad \alpha \in \mathbb{C}, \\
\mathfrak{D}(\tilde{\Delta})=\left\{f \in W_{2}^{2}\left(\mathbb{R}^{1} \backslash\left\{x_{1}\right\}\right) \mid f\left(x_{1}+\right)=f\left(x_{1}-\right), f^{\prime}\left(x_{2}+\right)-f^{\prime}\left(x_{2}-\right)=\alpha f\left(x_{1}\right)\right\} .
\end{gathered}
$$

In particular,

$$
\mathfrak{D}\left(-\tilde{\Delta}^{*}\right)=\left\{f \in W_{2}^{1}(\mathbb{R}) \cap W_{2}^{2}\left(\mathbb{R}^{1} \backslash\left\{x_{2}\right\}\right) \mid f\left(x_{2}+\right)=f\left(x_{2}-\right), f^{\prime}\left(x_{1}+\right)-f^{\prime}\left(x_{1}-\right)=\alpha f\left(x_{2}\right)\right\} .
$$

If $x_{1}=x_{2}$ and $\alpha \in \mathbb{R}$, then we obtain usual well-known case (1). Thus, we will investigate and study the construction and spectral properties of rank one singular perturbation of the form

$$
\tilde{A}=A+\alpha\left\langle\cdot, \omega_{1}\right\rangle \omega_{2}, \quad \omega_{1}, \omega_{2} \in \mathcal{H}_{-1} \backslash \mathcal{H},
$$

2010 Mathematics Subject Classification: 47A10, 47A55, 47A75, 34A37.

Keywords: rank one singular perturbation; eigenvalue problem; M. Krein's formula; nonselfadjoint perturbation; deviating argument.

doi:10.15330/ms.48.2.156-164

(C) T. I. Vdovenko, M. E. Dudkin, 2017 
where $A=A^{*}$ is a self-adjoint operator in the Hilbert space $\mathcal{H}, \mathcal{H}_{-1}$ is the space from the $A$-scale of spaces (see bellow) and $\alpha \in \mathbb{C} \cup \infty$.

Expecting spectral properties of the operator $-\tilde{\Delta}$ in (2) (also of $\tilde{A}$ in (3)) are different from $-\tilde{\Delta}$ in (1) because $\tilde{A}$ is a non self-adjoint operator in (3). But many standard facts of the singular perturbation theory of self-adjoint operators $([5,14])$ and their spectral properties have also corresponding analogies for expressions (2) and (3).

If $\omega_{1}=\omega_{2}$ and $\alpha \in \mathbb{R} \cup\{\infty\}$ in (3), then we obtain usual theory of the self-adjoint singular perturbations $([5,6,14])$. Hence, we obtain some natural generalization of the singular perturbation theory, considering expressions of form (3).

Our investigations have nontrivial intersection with a general considerations presented in [16]. In [16] authors considered a general object - the relation and its all proper extensions instead of one Hermitian operator. We also observe the special interest to such operators in [15]. In our investigations we meet really the pair of symmetric operators with defect indices $(1,1)$ both, and we consider only some class of non symmetric extensions. Presented investigations have partially joint ideas from [8], where perturbed normal operators were considered. The wave operators corresponding to (3) in case $\omega_{1}, \omega_{2} \in \mathcal{H}$ were actively investigated in [11]. Closely to $(2)$ related results $([18,19])$ are devoted to the non-local spectral problems (but in the case of self-adjoint perturbations).

The question: under what condition the Schrödinger operators have a point spectrum immersed in the continuous one, is difficult from a physical point of view. The study of this case is particularly unpromising, because there are good physical reasons to expect that such eigenvalues should not exist ([20]). However, there are known examples of J. von Neumann (1929) describing Hamiltonian perturbed by free smooth potentials such that a perturbed operator becomes coherent states inside the continuous spectrum. The main considerations for these cases mainly focused on how to avoid appearance of eigenvalues embedded in the continuous spectrum, as this creates difficulties in the scattering theory. But paper [3] contains the description of an unexpected example: rank one singularly perturbed self-adjoint operator possesses two new eigenvalues such that one of them is immersed in a continuous spectrum of the unperturbed (given) operator ([21]).

Since the study of singular perturbation operators is extended to perturbations by nonsymmetric potentials [9], you should expect also an associated pair of eigenvalues by rank one singularly perturbed non-symmetric potentials. In general, the paper is devoted to the description of an associated pair in our non-symmetric case.

There is a following advantage of approach ([3]): the proposed construction in [3] provides that the rank one singularly perturbed self-adjoint operator $\tilde{A}=A+\alpha\langle\cdot, \omega\rangle \omega$ possesses two new points in the point spectrum $\lambda, \mu \in \sigma_{p}(\tilde{A})$, i.e. $\tilde{A} \varphi_{\lambda}=\lambda \varphi_{\lambda}, \tilde{A} \varphi_{\mu}=\mu \varphi_{\mu}$, such that $\mu \in \rho(A)$ and $\lambda \in \sigma_{c}(A)$. Due to the construction we must choose arbitrary $\mu \in \rho(A)$, $\varphi_{\lambda} \in \mathcal{H} \backslash \mathfrak{D}(A)$ and calculate $\lambda$ and $\varphi_{\mu}$. Really, if we start to choose $\varphi_{\mu}$ so we can guarantee countable set of eigenvalues immersed in $\sigma_{c}(A)$ that is not so unexpected.

In fact, we investigate the inverse eigenvalue problem for perturbations of non-symmetric potentials. Namely, we present perturbation (3) which solves the eigenvalue problem for the dual pair: $\lambda, \mu \in \mathbb{C}$, such that $\tilde{A} \varphi_{\lambda}=\lambda \varphi_{\lambda}, \tilde{A} \varphi_{\mu}=\mu \varphi_{\mu}, \tilde{A}^{*} \psi_{\bar{\lambda}}=\bar{\lambda} \psi_{\bar{\lambda}}, \tilde{A}^{*} \psi_{\bar{\mu}}=\bar{\mu} \psi_{\bar{\mu}}$, $(\bar{\lambda}-\bar{\mu})\left((A-\mu)^{-1} \varphi_{\lambda}, \psi_{\bar{\lambda}}\right)=\left(\varphi_{\lambda}, \psi_{\bar{\lambda}}\right) \neq 0$.

Let us remark that if $\mu, \lambda \in \mathbb{R}$, then we obtain the case closely related to the self-adjoint one ([3]). But in a such case we can get a dual pair of eigenvalues with different eigenvectors corresponding to $\tilde{A}$ and $\tilde{A}^{*}$.

For realization of our ideas we use the mathematical tools from [9]. The tools in the case 
of self-adjoint operator $\left(\tilde{A}=\tilde{A}^{*}\right)$ were used in [2].

2. Preliminaries. Let $A=A^{*}$ be a self-adjoint unbounded operator defined on $\operatorname{Dom} A=$ $\mathfrak{D}(A)$ in a separable Hilbert space $\mathcal{H}$ with the inner product $(\cdot, \cdot)$ and the norm $\|\cdot\|$. Denote by $\sigma(\cdot), \sigma_{p}(\cdot), \sigma_{c}(\cdot), \rho(\cdot)$ spectrum, point spectrum, continuous spectrum and regular points of corresponding operator.

Definition 1 ([9]). The linear closed operator $\tilde{A} \neq A$ densely defined in $\mathcal{H}$ is call singularsingular perturbation $((s, s)$-perturbation) of $A$ if the sets

$$
\begin{aligned}
\mathfrak{D} & =\{f \in \mathfrak{D}(A) \cap \mathfrak{D}(\tilde{A}) \mid A f=\tilde{A} f\}, \\
\mathfrak{D}_{*} & =\left\{f \in \mathfrak{D}(A) \cap \mathfrak{D}\left(\tilde{A}^{*}\right) \mid A f=\tilde{A}^{*} f\right\}
\end{aligned}
$$

both are dense in $\mathcal{H}$. We denote $\tilde{A} \in \mathcal{P}_{s, s}(A)$.

Let us note that $\tilde{A}$ is a non self-adjoint operator in general.

It is clear that for each operator $\tilde{A} \in \mathcal{P}_{s, s}(A)$, there exists a pair of densely defined restricted symmetric operators $\dot{A}:=A \uparrow \mathfrak{D}$ and $\dot{A}_{*}:=A \uparrow \mathfrak{D}_{*}$ with nontrivial deficiency indices $\mathbf{n}^{ \pm}(\dot{A})=\operatorname{dim} \operatorname{ker}(\dot{A} \mp z)^{*} \neq 0$, and $\mathbf{n}^{ \pm}\left(\dot{A}_{*}\right)=\operatorname{dim} \operatorname{ker}\left(\dot{A}_{*} \mp z\right)^{*} \neq 0, z \in \rho(A)$. In this article we suppose that $\mathbf{n}^{ \pm}(\dot{A})=\mathbf{n}^{ \pm}\left(\dot{A}_{*}\right)=1$, i.e. we discuss the case $\tilde{A} \in \mathcal{P}_{s, s}^{1,1}(A)$.

If $\mathfrak{D}=\mathfrak{D}_{*}$ and $\tilde{A}=\tilde{A}^{*}$, then we obtain usual abstract definition of the singularly perturbed self-adjoint operators $([5,14]) \tilde{A} \in \mathcal{P}_{s}(A)$, i.e. Definition 1 generalizes the known definition of the singular self-adjoint perturbation on the case of non self-adjoint one.

By $\left\{\mathcal{H}_{k}(A)\right\}_{k \in \mathbb{R}^{1}}$ we denote the associated $A$-scale of Hilbert spaces $([5])$, where the space $\mathcal{H}_{k}:=\mathcal{H}_{k}(A)=\mathfrak{D}\left(|A|^{k / 2}\right), k \geq 1$ with the norm $\|\varphi\|_{k}=\left\|(|A|+I)^{k / 2} \varphi\right\|$ (and $I$ stands for the identity) $\varphi \in \mathcal{H}_{k}(A)$ and $\mathcal{H}_{-k}:=\mathcal{H}_{-k}(A)$ is a negative (dual) space i.e. the completion of $\mathcal{H}$ in the norm $\|f\|_{-k}=\left\|(|A|+I)^{-k / 2} f\right\|, f \in \mathcal{H}$. Let $\langle\cdot, \cdot\rangle$ be a dual dot product between the spaces $\mathcal{H}_{k}$ and $\mathcal{H}_{-k}$. For the next consideration, really, we take only $k=1,2$.

The operator $A$ can be continued on $\mathcal{H}_{+1}$ and it is understanding as the bounded operator from $\mathcal{H}_{+1}$ into $\mathcal{H}_{-1}$. (Analogously $A$ can be continued on $\mathcal{H}$ and understanding as the bounded operator from $\mathcal{H}$ on the whole $\mathcal{H}_{-2}$.) We denote this continuation by $\mathbf{A}$. Therefore, the expression $\langle\varphi, \omega\rangle$ for $\omega=\mathbf{A} \psi$ has the sense with $\varphi, \psi \in \mathcal{H}_{+1}$. And $\mathbf{R}_{z}=(\mathbf{A}-z)^{-1}$, $z \in \rho(A)$ is the corresponding resolvent.

Since the vectors $\omega_{1}, \omega_{2}$ in $(3)$ can belong to $\mathcal{H}_{-k} \backslash \mathcal{H}_{-(k-1)}, k=0,1,2$, then we distinguish different arts of (especially regular) perturbation. For this reason we denote $\mathcal{P}_{x, y}(A)$, where each index of the couple $\{x, y\}$ can possess one of the symbol "ss, ws, $r$ ", that means "strong singular" and "weakly singular" and "regular" vector of perturbation. The symbol " $s$ " is reserved for one of two cases — "strong singular" and "weakly singular" perturbation. After previous description we give a definition.

Definition 2. If for the sets $\mathcal{D}$ and $\mathcal{D}_{*}$ in (4) and (5) we have

$$
\begin{aligned}
\operatorname{dim}(\mathcal{H} \ominus \mathfrak{D})=0, & \operatorname{dim}\left(\mathcal{H}_{+1} \ominus \mathfrak{D}\right)=1(=n \neq 0), \\
\operatorname{dim}\left(\mathcal{H} \ominus \mathfrak{D}_{*}\right)=0, & \operatorname{dim}\left(\mathcal{H}_{+1} \ominus \mathfrak{D}_{*}\right)=1(=m \neq 0),
\end{aligned}
$$

then the perturbation is called "weakly-weakly" singular, i.e. (ws,ws)-singular of rank oneone (i.e. $(1,1))$ and is denoted by $\tilde{A} \in \mathcal{P}_{w s, w s}^{1,1}(A)$ and for $\operatorname{rank}(n, m):\left(\tilde{A} \in \mathcal{P}_{w s, w s}^{n, m}(A)\right)$. 
If for the sets $\mathcal{D}$ and $\mathcal{D}_{*}$ in (4) and (5) we have

$$
\begin{aligned}
& \operatorname{dim}\left(\mathcal{H}_{+1} \ominus \mathfrak{D}\right)=0, \operatorname{dim}\left(\mathcal{H}_{+2} \ominus \mathfrak{D}\right)=1(=n \neq 0), \\
& \operatorname{dim}\left(\mathcal{H}_{+1} \ominus \mathfrak{D}_{*}\right)=0, \quad \operatorname{dim}\left(\mathcal{H}_{+2} \ominus \mathfrak{D}_{*}\right)=1(=m \neq 0),
\end{aligned}
$$

then the perturbation is called "strong-strong" singular, i.e. $(s s, s s)$-singular of rank one-one (i.e. $(1,1))$ and is denoted by $\tilde{A} \in \mathcal{P}_{s s, s s}^{1,1}(A)$ and for $\operatorname{rank}(n, m):\left(\tilde{A} \in \mathcal{P}_{s s, s s}^{n, m}(A)\right)$.

If for the sets $\mathcal{D}$ and $\mathcal{D}_{*}$ in (4) and (5) we have

$$
\operatorname{dim}(\mathcal{H} \ominus \mathfrak{D})=1(=n \neq 0), \quad \operatorname{dim}\left(\mathcal{H} \ominus \mathfrak{D}_{*}\right)=1(=m \neq 0),
$$

then the perturbation is called "regular-regular", i.e. $(r, r)$-perturbation of rank one-one (i.e. $(1,1))$ and is denoted by $\tilde{A} \in \mathcal{P}_{r, r}^{1,1}(A)$ and for $\operatorname{rank}(n, m):\left(\tilde{A} \in \mathcal{P}_{r, r}^{n, m}(A)\right)$.

Of course, there may be mixed cases. Perturbations of mixed type $(s s, w s),(s s, r),(w s, r)$, i.e. $\mathcal{P}_{s s, w s}(A), \mathcal{P}_{s s, r}(A)$ and $\mathcal{P}_{w s, r}(A)$, as also cases $n>1, m>1$ in this publication are not considered.

Here we consider only the case "weakly-weakly" singular perturbation of rank one-one, i.e. we investigate the spectral properties of the operator $\tilde{A} \in \mathcal{P}_{\text {ws ws }}^{1,1}(A)$. Another types of perturbation will be considered in the next publications, since another cases need some special methods.

Let us consider in $A$-scale an operator $V$ such that $\mathfrak{D}(V) \subseteq \mathcal{H}_{+1}$ and $\mathcal{R}(V) \subseteq \mathcal{H}_{-1}$. In our case $V=\alpha\left\langle\cdot, \omega_{1}\right\rangle \omega_{2}$ (compare with (3)). We define the operator $A+V$ as the sum

$$
(A+V) \varphi:=(\mathbf{A} \varphi+V \varphi), \quad \varphi \in \mathfrak{D}(A+V):=\{\varphi \in \mathfrak{D}(V) \mid \mathbf{A} \varphi+V \varphi \in \mathcal{H}\},
$$

shortly $\tilde{A} f=\left(\mathbf{A} f+\alpha\left\langle f, \omega_{1}\right\rangle \omega_{2}\right) \uparrow \mathcal{H}, f \in \mathcal{H}_{+1}$. Below without loss of understanding we usually write $A$ instead of $\mathbf{A}$ and $R_{z}$ instead of $\mathbf{R}_{z}$.

Let us present the particular definition of $\tilde{A} \in \mathcal{P}_{w s, w s}^{1,1}(A)$, which follows directly from Definitions 1 and 2 .

Definition $3([7])$. The operator $\tilde{A}$ is called a rank one non-symmetric weakly singular perturbation of a self-adjoint operator $A$ in a separable Hilbert space $\mathcal{H}$, if for $\eta_{i}=\mathbf{A}^{-1} \omega_{i}$, such that $\omega_{1} \in \mathcal{H}_{-1} \backslash \mathcal{H}$, or $\omega_{2} \in \mathcal{H}_{-1} \backslash \mathcal{H}, \omega_{1} \neq \omega_{2}$ :

$$
\mathfrak{D}(\tilde{A})=\left\{\psi=\varphi+b \eta_{2} \mid \varphi \in \mathfrak{D}(A), b=\frac{\left(A \varphi, \eta_{1}\right)}{1+\left(A^{1 / 2} \eta_{2}, A^{1 / 2} \eta_{1}\right)}\right\}
$$

in the case $\left(A^{1 / 2} \eta_{2}, A^{1 / 2} \eta_{1}\right) \neq-1$; and

$$
\mathfrak{D}\left(A^{\omega_{1}, \omega_{2}}\right)=\mathfrak{D}_{\mathcal{H}_{1}} \dot{+}\{c \eta\}, \mathfrak{D}_{\mathcal{H}_{1}}=\left\{\varphi \in \mathfrak{D}(A) \mid\left(A \varphi, \eta_{1}\right)=0\right\}
$$

in the case $\left(A^{1 / 2} \eta_{2}, A^{1 / 2} \eta_{1}\right)=-1$; and the action is defined by the rule: $A^{\omega_{1}, \omega_{2}} \psi=A \varphi$.

3. The description and spectral properties of the rank one singular non-symmetric perturbations. We remark that if $\tilde{A} \in \mathcal{P}_{w s, w s}(A)$ then for the adjoint operator we have $\tilde{A}^{*} \in \mathcal{P}_{w s, w s}(A)$..

Proposition 1. Each operator $\tilde{A} \in \mathcal{P}_{w s, w s}^{1,1}(A)$ (Definitions 1 and 2 or 3 ) admits the representation in the form

$$
\tilde{A}=A+\alpha\left\langle\cdot, \omega_{1}\right\rangle \omega_{2},
$$

with $\alpha \in \mathbb{C} \cup\{\infty\}$ and $\omega_{1}, \omega_{2} \in \mathcal{H}_{-1} \backslash \mathcal{H}$. 
The next theorem describes the rank one singular non-symmetric perturbations by the resolvent form of corresponding operator.

Theorem $1([9])$. For the resolvents $R_{z}=(A-z)^{-1}$ and $\tilde{R}_{z}=(\tilde{A}-z)^{-1}$ of operators $A=A^{*}$ and $\tilde{A} \in \mathcal{P}_{w s, w s}^{1,1}(A)$ in the separable Hilbert space $\mathcal{H}$ one can write the like $M$. Krein formula for $z, \xi, \zeta \in \rho(A) \cap \rho(\tilde{A})$

$$
\tilde{R}_{z}=R_{z}+b_{z}\left(\cdot, n_{\bar{z}}\right) m_{z}
$$

with

$$
n_{z}=(A-\xi)(A-z)^{-1} n_{\xi}, \quad m_{z}=(A-\zeta)(A-z)^{-1} m_{\zeta},
$$

where $n_{z}, m_{z} \in \mathcal{H}_{+1} \backslash \mathcal{H}_{+2}$ and

$$
b_{z}^{-1}-b_{\xi}^{-1}=(\xi-z)\left(m_{\xi}, n_{\bar{z}}\right) .
$$

The vectors $n_{z}, m_{z}$ and the number $b_{z}$ are connected with $\omega_{1}, \omega_{2}$ from (7) as follows

$$
n_{z}=R_{z} \omega_{1}, m_{z}=R_{z} \omega_{2}, \quad-b_{z}^{-1}=\alpha^{-1}+\left\langle\omega_{2}, R_{\bar{z}} \omega_{1}\right\rangle
$$

where $\alpha \neq 0$.

We can include the case $\alpha=0$ into consideration, since if $\alpha=0$, one can put $b_{z} \equiv 0$ and have $\tilde{R}_{z} \equiv R_{z}$.

Possibly $b_{z}=\infty$, and it is so if and only if $z \in \sigma_{p}(\tilde{A})$, but (8) is also valid for such case.

The continuous spectrum $\sigma_{c}(A)$ of the operator $A$ under the finite rank perturbations is unchangeable i.e. $\sigma_{c}(A)=\sigma_{c}(\tilde{A}), \tilde{A} \in \mathcal{P}_{s, s}^{n, n}(A), n<\infty$.

Theorem 2. Let $\tilde{A} \in \mathcal{P}_{w s, w s}^{1,1}(A)$ be given in the form (8) with (9) and (10) possess a new eigenvalue $\lambda \in \mathbb{C}$ in compare with $A$ i.e. there exists $\lambda \in \sigma_{p}(\tilde{A}), \lambda \notin \sigma_{p}(A)$, then for corresponding eigenvectors $\varphi, \psi: \tilde{A} \varphi=\lambda \varphi$ and $\tilde{A}^{*} \psi=\bar{\lambda} \psi$ the following relations hold

$$
\begin{aligned}
& (\lambda-z) b_{z}\left(\varphi, n_{\bar{z}}\right)=1, \quad \varphi=(A-z)(A-\lambda)^{-1} m_{z} \\
& (\bar{\lambda}-\bar{z}) \bar{b}_{z}\left(\psi, m_{z}\right)=1, \quad \psi=(A-\bar{z})(A-\bar{\lambda})^{-1} n_{\bar{z}} .
\end{aligned}
$$

Proof. Using the equality $\tilde{A} \varphi=\lambda \varphi$ i.e. $R_{z} \varphi+b_{z}\left(\varphi, n_{\bar{z}}\right) m_{z}=\frac{1}{\lambda-z} \varphi$, we get

$$
(\lambda-z) b_{z}\left(\varphi, n_{\bar{z}}\right)(A-z)(A-\lambda)^{-1} m_{z}=\varphi
$$

that gives (11).

Analogously considering $\tilde{A}^{*} \psi=\bar{\lambda} \psi$ we can prove (12).

Analogously to Theorem 2 where $\tilde{A}$ is given by (8) we have the next corollary where $\tilde{A}$ is given by (7).

Corollary 1. Let $\tilde{A} \in \mathcal{P}_{w s, w s}^{1,1}(A)$ possesses new eigenvalue $\lambda \in \mathbb{C}$ in compare with $A$ and eigenvectors $\varphi$ and $\psi$ i.e. $\tilde{A} \varphi=\lambda \varphi$ and $\tilde{A}^{*} \psi=\bar{\lambda} \psi$, then relations (11) and (12) in terms of $\omega_{1}, \omega_{2}$ have a form:

$$
\begin{aligned}
\alpha\left\langle(A-\lambda)^{-1} \omega_{2}, \omega_{1}\right\rangle & =-1, \quad \varphi=(A-\lambda)^{-1} \omega_{2} \\
\bar{\alpha}\left\langle(A-\bar{\lambda})^{-1} \omega_{1}, \omega_{2}\right\rangle & =-1, \quad \psi=(A-\bar{\lambda})^{-1} \omega_{1} .
\end{aligned}
$$


We will call the formulation of Theorem 2 as direct spectral problem, one can consider the inverse problem in the next theorem.

Theorem 3. For a given self-adjoint operator $A=A^{*}$ in a separable Hilbert space $\mathcal{H}$ and $\lambda \in \mathbb{C}$ and vectors $\varphi, \psi \in \mathcal{H}_{+1} \backslash \mathcal{H}_{+2}$, such that $\langle(A-\bar{\lambda}) \varphi, \psi\rangle \neq 0$, there exists unique $\tilde{A} \in \mathcal{P}_{w s, w s}^{1,1}(A)$ such that $\tilde{A} \varphi=\lambda \varphi$ and $\tilde{A}^{*} \psi=\bar{\lambda} \psi$.

The operator $\tilde{A}$ is defined by (8) as follows

$$
\tilde{R}_{z}=R_{z}+b_{z}\left(\cdot, n_{\bar{z}}\right) m_{z}
$$

with

$$
m_{z}=(A-\lambda)(A-z)^{-1} \varphi, \quad n_{\bar{z}}=(A-\bar{\lambda})(A-\bar{z})^{-1} \psi
$$

and

$$
b_{z}^{-1}=(\lambda-z)\left(\varphi, n_{\bar{z}}\right), \quad\left(\bar{b}_{z}^{-1}=(\bar{\lambda}-\bar{z})\left(\psi, m_{z}\right)\right) .
$$

Theorems $1-3$ in case of the self-adjoint perturbed operator $\tilde{A}=\tilde{A}^{*}$ are proved in $[2,10]$.

4. Dual pair of eigenvalues. Since $\tilde{A} \in \mathcal{P}_{w s, w s}^{1,1}(A)$ in general is non self-adjoint operator then the definition of dual pair of eigenvalues is different from [3].

Definition 4. The couple of numbers $\lambda, \mu \in \mathbb{C}$ is called a dual pair of eigenvalues of singularly perturbed operator $\tilde{A} \in \mathcal{P}_{w s, w s}^{1,1}(A)$, if

$$
\begin{gathered}
\tilde{A} \varphi_{\lambda}=\lambda \varphi_{\lambda}, \tilde{A} \varphi_{\mu}=\mu \varphi_{\mu}, \tilde{A}^{*} \psi_{\bar{\lambda}}=\bar{\lambda} \psi_{\bar{\lambda}} \\
\tilde{A}^{*} \psi_{\bar{\mu}}=\bar{\mu} \psi_{\bar{\mu}},(\bar{\lambda}-\bar{\mu})\left((A-\mu)^{-1} \varphi_{\lambda}, \psi_{\bar{\lambda}}\right)=\left(\varphi_{\lambda}, \psi_{\bar{\lambda}}\right) \neq 0 .
\end{gathered}
$$

The next theorem describes the method of construction of the dual pair.

Theorem 4. Let $A=A^{*}$ be a self-adjoint operator defined on $\mathfrak{D}(A)$ in a separable Hilbert space $\mathcal{H}$. For an arbitrary fixed $\mu \in \rho(A)$ and vectors $\varphi_{\lambda}, \psi_{\bar{\lambda}} \in \mathcal{H}_{+1} \backslash \mathcal{H}_{+2}$, such that $\left(\varphi_{\lambda}, \psi_{\bar{\lambda}}\right) \neq 0$, there exists an unique non-symmetric singularly perturbed operator $\tilde{A} \in$ $\mathcal{P}_{w s, w s}^{1,1}(A)$, such that $(\mu, \lambda)$ is dual pair, where $\bar{\lambda}:=\bar{\mu}+\frac{\left(\varphi_{\lambda}, \psi_{\bar{\lambda}}\right)}{\left((A-\mu)^{-1} \varphi_{\lambda}, \psi_{\bar{\lambda}}\right)}$ is an eigenvalue with the eigenvector $\varphi_{\mu}=(A-\lambda)(A-\mu)^{-1} \varphi_{\lambda}$.

The operator $\tilde{A}^{*} \in \mathcal{P}_{w s, w s}^{1,1}(A)$ has eigenvectors $\psi_{\bar{\lambda}}, \psi_{\bar{\mu}}=(A-\bar{\lambda})(A-\bar{\mu}) \psi_{\bar{\lambda}}$.

If the operator $\tilde{A}$ is given in the form $\tilde{A}=A+\alpha\left\langle\cdot, \omega_{1}\right\rangle \omega_{2}$, then it has the coupling constant $\alpha=-\frac{1}{\left(\varphi_{\lambda}, \omega_{1}\right)}\left(\right.$ or $\left.\bar{\alpha}=-\frac{1}{\left(\psi_{\bar{\lambda}}, \omega_{2}\right)}\right)$ with corresponding vectors

$$
\omega_{2}=(A-\mu) \varphi_{\lambda}-\frac{\left(\psi_{\bar{\lambda}}, \varphi_{\lambda}\right)}{\left((A-\mu) \psi_{\bar{\lambda}}, \varphi_{\lambda}\right)} \varphi_{\lambda}, \quad \omega_{1}=(A-\bar{\mu}) \psi_{\bar{\lambda}}-\frac{\left(\varphi_{\lambda}, \psi_{\bar{\lambda}}\right)}{\left((A-\mu) \varphi_{\lambda}, \psi_{\bar{\lambda}}\right)} \psi_{\bar{\lambda}} .
$$

Proof. We start with the proof of Theorem 4. For an arbitrary $\mu \in \rho(A)$ and $\varphi=\varphi_{\lambda} \in$ $\mathcal{H}_{+1} \backslash \mathfrak{D}(A), \psi=\psi_{\bar{\lambda}} \in \mathcal{H}_{+1} \backslash \mathfrak{D}(A)$ we put

$$
\bar{\lambda}=\bar{\mu}+\frac{\left(\varphi_{\lambda}, \psi_{\bar{\lambda}}\right)}{\left((A-\mu)^{-1} \varphi_{\lambda}, \psi_{\bar{\lambda}}\right)} .
$$


In Theorem 3 there exists an unique operator $\tilde{A} \in \mathcal{P}_{\text {ws,ws }}^{1,1}(A)$, such that $A \varphi_{\lambda}=\lambda \varphi_{\lambda}$ and $\tilde{A}^{*} \psi_{\bar{\lambda}}=\bar{\lambda} \psi_{\bar{\lambda}}$ given by resolvent form (8): $\tilde{R}_{z}=R_{z}+b_{z}\left(\cdot, n_{\bar{z}}\right) m_{z}$, with (16) and (17):

$$
m_{z}=(A-\lambda)^{-1}(A-z)^{-1} \varphi_{\lambda}, n_{\bar{z}}=(A-\bar{\lambda})^{-1}(A-\bar{z})^{-1} \varphi_{\bar{\lambda}}, b_{z}=\frac{1}{(\lambda-z)\left(\varphi, n_{\bar{z}}\right)} .
$$

The factor $(\lambda-z)$ in the denominator of the last expression shows that $\lambda$ is an eigenvalue.

The second eigenvalue can be obtained from the fact that the second factor in the denominator can be equals zero

$$
0=\left(\varphi, n_{\bar{z}}\right)=\left(\varphi_{\lambda},\left(\psi_{\bar{\lambda}}+(\bar{z}-\bar{\lambda})(A-\bar{z})^{-1} \psi_{\bar{\lambda}}\right),\right.
$$

where from Theorem $3 n_{\bar{z}}=(A-\lambda)(A-\bar{z})^{-1} \psi_{\bar{\lambda}}$. Hence, $\left(\varphi_{\lambda}, \psi_{\bar{\lambda}}\right)=(\bar{\lambda}-\bar{z})\left((A-z)^{-1} \varphi_{\lambda}, \psi_{\bar{\lambda}}\right)$ and we obtain (19). Replacing $z$ on $\mu$ in (20) we obtain $\varphi_{\mu}=m_{\mu}=(A-\lambda)(A-\mu)^{-1} \varphi_{\lambda}$. Particularly, $\varphi_{\lambda} \perp \psi_{\bar{\mu}}$.

Analogously using $\left(A^{*}-z\right)^{-1}=R_{z}+b_{z}\left(\cdot, m_{z}\right) n_{\bar{z}}$ for the operator $\tilde{A}^{*} \in \mathcal{P}_{w s, w s}^{1,1}(A)$, we obtain $\tilde{A}^{*} \varphi_{\bar{\lambda}}=\lambda \varphi_{\bar{\lambda}}$ and $\tilde{A}^{*} \psi_{\bar{\mu}}=\bar{\mu} \psi_{\bar{\mu}}$.

For the real dual pair $\lambda, \mu \in \mathbb{R}$ we have the following corollary from Theorem 4 .

Corollary 2. Let $A=A^{*} \geq 0$ be a positive self-adjoint operator defined on $\mathfrak{D}(A)$ in a separable Hilbert space $\mathcal{H}$ such that $\sigma(A)=\sigma_{c}(A)=[0, \infty)$. For an arbitrary number $\mu<0$ and vectors $\varphi_{\lambda}, \psi_{\bar{\lambda}} \in \mathcal{H}_{+1} \backslash \mathcal{H}_{+2}$ such that $\left(\varphi_{\lambda}, \psi_{\bar{\lambda}}\right) \neq 0$, there exists an unique non-symmetric singular perturbation of rank one $\tilde{A} \in \mathcal{P}_{w s, w s}^{1,1}(A)$ such that has dual pair $(\mu, \lambda)$, where $\lambda=\mu+\frac{\left(\varphi_{\lambda}, \psi \lambda\right)}{\left((A-\mu)^{-1} \varphi_{\lambda}, \psi_{\lambda}\right)}$ are its eigenvalues with eigenvector $\varphi_{\lambda}$ and $\varphi_{\mu}=(A-\lambda)(A-\mu)^{-1} \varphi_{\lambda}$.

The operator $\tilde{A}^{*} \in \mathcal{P}_{w s, w s}^{1,1}(A)$ has the same eigenvalues with eigenvectors $\psi_{\lambda}$ and $\psi_{\mu}=$ $(A-\lambda)(A-\mu) \psi_{\lambda}$. In particular $\lambda \in(0, \infty)$.

\section{Examples.}

Example 1. Let $\mathcal{H}=L_{2}([1, \infty), d x)$ and $A$ be the multiplication operator of the independent variable $x$, i.e. $A f(x)=x f(x), f \in \mathfrak{D}(A)$, where $\mathfrak{D}(A):=\left\{f(x) \in L_{2} \mid x f(x) \in L_{2}\right\}$. Obviously, $A \geq 1$ and $\mathfrak{D}(A)=\sigma(A)=[1, \infty)$. Let us put $0=\mu \notin \sigma(A)$ and $\varphi=\varphi_{\lambda}=x^{-1 \frac{1}{3}}$, $\psi=\psi_{\lambda}=x^{-1 \frac{2}{3}}, \varphi, \psi \in \mathcal{H}_{+1}=L_{2}([1, \infty), x d x)$. Thus, $\mathcal{H}_{+2}=L_{2}\left([1, \infty), x^{2} d x\right)$ and $\varphi, \psi \notin$ $\mathcal{H}_{+2}$, then

$$
(\varphi, \psi)=\int_{1}^{\infty} \frac{d x}{x^{3}}=\frac{1}{2}, \quad\left((A-\mu)^{-1} \varphi, \psi\right)=\int_{1}^{\infty} \frac{d x}{x^{4}}=\frac{1}{3} .
$$

Hence, $\lambda=\frac{3}{2} \in \sigma(A)$; and

$$
\varphi_{\mu}=\left(A-\frac{3}{2}\right) A^{-1} \varphi_{\lambda}=\frac{x-\frac{3}{2}}{x^{7 / 3}}, \quad \psi_{\mu}=\left(A-\frac{3}{2}\right) A^{-1} \psi_{\lambda}=\frac{x-\frac{3}{2}}{x^{8 / 3}}
$$

And from (16) it follows that

$$
m_{z}=(A-\lambda)(A-z)^{-1} \varphi_{\lambda}=\frac{x-3 / 2}{x-z} \frac{1}{x^{4 / 3}}, \quad n_{z}=(A-\bar{\lambda})(A-\bar{z})^{-1} \psi_{\lambda}=\frac{x-3 / 2}{x-z} \frac{1}{x^{5 / 3}},
$$

and from (17) we have: $b_{z}=(\lambda-z)^{-1}\left(\varphi_{\lambda}, n_{\bar{z}}\right)^{-1}=\left(\frac{3}{2}-z\right)^{-1}\left(\varphi_{\lambda}, n_{\bar{z}}\right)^{-1}$, where

$$
\left(\varphi_{\lambda}, \eta_{\bar{z}}\right)=\int_{1}^{\infty} \frac{1}{x^{11 / 3}} \frac{x-3 / 2}{x-z} \frac{1}{x^{1 / 3}} d x=\left(\frac{3}{2 z^{3}}-\frac{1}{z^{2}}\right) \ln (1-z)+\left(\frac{3}{2 z^{2}}-\frac{1}{z}\right)+\frac{3}{4 z} .
$$


Hence, (15) has form

$$
(\tilde{A}-z)^{-1}=\frac{1}{x-z}+b_{z}\left(\cdot \frac{x-3 / 2}{x-\bar{z}} \frac{1}{x^{5 / 3}}\right) \frac{x-3 / 2}{x-z} \frac{1}{x^{4 / 3}} .
$$

Furthermore,

$$
\begin{gathered}
\omega_{1}=\frac{x}{x^{5 / 3}}-\frac{1 / 2}{1 / 3} \frac{1}{x^{5 / 3}}=\frac{2 x-3}{2 x^{5 / 3}}, \quad \omega_{2}=\frac{x}{x^{4 / 3}}-\frac{1 / 2}{1 / 3} \frac{1}{x^{4 / 3}}=\frac{2 x-3}{2 x^{4 / 3}} \\
\left\langle\varphi_{\lambda}, \omega_{1}\right\rangle=\int_{1}^{\infty} \frac{1}{x^{4 / 3}} \frac{2 x-3}{2 x^{5 / 3}} d x=\left\langle\psi_{\lambda}, \omega_{2}\right\rangle=\int_{1}^{\infty} \frac{1}{x^{5 / 3}} \frac{x-3}{2 x^{4 / 3}} d x=\frac{1}{2} \int_{1}^{\infty} \frac{2 x-3}{x^{3}} d x=1 / 4 .
\end{gathered}
$$

Taking into account (13), (14), we deduce $\alpha=-\frac{1}{\left\langle\varphi_{\lambda}, \omega_{1}\right\rangle}=-4$.

Example 2. Let $\mathcal{H}=L_{2}\left(\mathbb{R}^{1}, d x\right)$ and $A$ plays the role of the Laplace operator, namely $A f(x)=-f^{\prime \prime}(x), \mathfrak{D}(A)=W_{2}^{2}\left(\mathbb{R}^{1}\right)$ is a Sobolev space. The operator $A \geq 0$ has purely continuous spectrum i.e. $\sigma(A)=\sigma_{c}(A)=[0, \infty)$.

Let us put $\varphi_{\lambda}=e^{-|x-1|}$ and $\psi_{\lambda}=e^{-|x+1|}, \mu=-1$ (we consider the case $\lambda, \mu \in \mathbb{R}$ and $\lambda, \mu \in \rho(A))$. For calculating $\lambda$ we need $\left(\varphi_{\lambda}, \psi_{\lambda}\right)=\int_{\mathbb{R}} e^{-|x-1|} e^{-|x+1|} d x=3 e^{-2}$. Using [1], we deduce

$$
(A+1)^{-1} \varphi_{\lambda}=\int_{\mathbb{R}} \frac{1}{2} e^{-|x-\tau|} e^{-|\tau-1|} d \tau= \begin{cases}\frac{x}{2} e^{-x+1}, & x>1, \\ \frac{2-x}{2} e^{x-1}, & x<1,\end{cases}
$$

and consequently $\left((A+1)^{-1} \varphi_{\lambda}, \psi_{\lambda}\right)=\frac{13}{4} e^{-2}$. Hence, $\lambda=-\frac{1}{13}<0, \alpha=-\frac{4}{13} e^{2}$. And from (18) we have $([21])$

$$
\omega_{1}=\delta_{-1}(x)-\frac{12}{13} e^{-|x+1|}, \quad \omega_{2}=\delta_{+1}(x)-\frac{12}{13} e^{-|x-1|} .
$$

Example 3. We slightly modify the previous example. Namely, we leave $\mathcal{H}=L_{2}\left(\mathbb{R}^{1}, d x\right)$ and the operator $A f(x)=-f^{\prime \prime}(x), \mathfrak{D}(A)=W_{2}^{2}\left(\mathbb{R}^{1}\right)$, but choose $\varphi_{\lambda}=e^{-|x-1|}$ and $\psi_{\lambda}=e^{-|x|}$, $\mu=-1$ (we also consider the case $\lambda, \mu \in \mathbb{R}$, but $\lambda \in \sigma(A), \mu \in \rho(A)$ ). To calculate $\lambda$ we need

$$
\left(\varphi_{\lambda}, \psi_{\lambda}\right)=\int_{\mathbb{R}} e^{-|x-1|} e^{-|x|} d x=2 e^{-1} .
$$

Using (21) we have $\left((A+1)^{-1} \varphi_{\lambda}, \psi_{\lambda}\right)=\frac{7}{4} e^{-1}$. Hence, $\lambda=\frac{1}{15}>0, \alpha=-\frac{1}{7} e$. And from (18) we have $([21]) \omega_{1}=\delta_{0}(x)-\frac{8}{7} e^{-|x|}, \omega_{2}=\delta_{+1}(x)-\frac{8}{7} e^{-|x-1|}$.

We can interpret expression (2) for applications as " $\delta$-interaction with a retarding" if $x_{1}<x_{2}$ that has a physical sense in the deviating differential models. With this connection, the case $x_{1}>x_{2}$ we can understand as a "ahead potential".

The operators (3) appear in the delay differential equations i.e. equations with retarded potentials, equations that has functions with deviating (linearly transformed) argument (see a general theory in $[13,17])$.

The presented methods in the paper permit us to consider the corresponding SturmLiouville type equations. 


\section{REFERENCES}

1. S. Albeverio, F. Gesztesy, R. Høegh-Krohn, H. Holden, Solvable models in quantum mechanics. Second edition, With an appendix by Pavel Exner, AMS Chelsea Publishing, Providence, RI, 2005.

2. S. Albeverio, M. Dudkin, A. Konstantinov, V. Koshmanenko, On the point spectrum of $H_{-2}$-singular perturbations, Math. Nachr., 280 (2007), №1-2, 20-27.

3. S. Albeverio, M. Dudkin, V. Koshmanenko, Dual pair of eigenvalues, Letters in Math. Phis., 63 (2003), 219-228.

4. S. Albeverio, R. Hryniv, L. Nizhnik Inverse spectral problems for nonlocal Sturm-Liouville operators, Inverse Problems, 23 (2007), 523-535.

5. S. Albeverio, P. Kurasov, Singular perturbations of differential operators. Solvable Schrödinger type operators, London Mathematical Society Lecture Note Series, 271, Cambridge University Press, Cambridge, 2000.

6. Y. Berezansky, J. Brasche, Generalized selfadjoint operators and their singular perturbations, Methods Funct. Anal. Topology, 7 (2001), №3, 54-66.

7. M.E. Dudkin, T.I. Vdovenko, Syngular rank one non-symmetric perturbations of a self-adjoint operator. in "The spectral theorey of operators and sets of operators", Proceedings of Institute of Mathematics of NAS of Ukraine, 12 (2015), №1, 57-73. (in Ukrainian)

8. M.E. Dudkin, Singularly perturbed rank one normal operators and its applications, Preprint, Institute of Mathematics NAS of Ukraine, 2008, 38 p.

9. M.E. Dudkin, T.I. Vdovenko, Rank one strong singular perturbation by non-symmetric potential, Research Bulletin of National Technical Univ. of Ukraine "KPI", 96 (2014), 13-17.

10. M.E. Dudkin, V.D. Koshmanenko, The point spectrum of self-adjoint operators that appears under singular perturbations of finite rank, Ukrainian Math. J., 55 (2003), №9, 1532-1541.

11. T. Kato, Wave operators and similarity for some non self-adjoint operators, Math. Annalen, 162 (1966), 258-279.

12. T. Kato, Perturbation theory for linear operators, Reprint of the 1980 edition. Classics in Mathematics, Springer-Verlag, Berlin, 1995.

13. T. Kato, J.B. McLeod, The functional-differential equation $y^{\prime}(x)=a y(\lambda x)+b y(x)$, Bull. Amer. Math. Soc., 77 (1971), 891-937.

14. V. Koshmanenko, Singular quadratic forms in perturbation theory, Translated from the 1993 Russian original by P. V. Malyshev and D. V. Malyshev, Mathematics and its Applications, 474., Kluwer Academic Publishers, Dordrecht, 1999.

15. V.B. Lidskii, The non self-adjoint operator of Sturm-Liouville type with discrete spectrum, Trudy Moskow. Mat. Obshchestva, 9 (1960), 45-79. (in Russian)

16. M.M. Malamud, V.I. Mogilevskii, Kreı̆n type formula for canonical resolvents of dual pairs of linear relations, Methods Funct. Anal. Topology, 8 (2002), №4, 72-100.

17. A.D. Myshkis, General theory of differential equations with retarded arguments, Uspekhi Mat. Nauk, 4 (1949), №5(33), 99-141. (in Russian)

18. L. Nizhnik, Inverse nonlocal Sturm-Liouville problem, Inverse problems, 26, (2010), 9 p.

19. L. Nizhnik, Inverse spectral nonlocal problem for the first order ordinary differential equation, Tamkang Journal of Mathematics, 42 (2011), №3, 385-394.

20. M. Reed, B. Simon, Methods of modern mathematical physics. IV. Analysis of operators, Academic Press, New York-San Francisco-London, 1978.

21. T.I. Vdovenko, The couple of eigenvalues of non-symmetric rank one singularly perturbed operators, Naukovi zapysky NaUKMA, 178 (2016), 3-8. (in Ukrainian)

National Technical University of Ukraine, Kyiv

tanyavdovenko@meta.ua

dudkin@imath.kiev.ua 